\title{
Interactive Decision Making for Multiobjective Bimatrix Games with Fuzzy Payoffs Based on Possibility Measure
}

\author{
${ }^{*}$ Hitoshi Yano ${ }^{a}$ and Ichiro Nishizaki ${ }^{b}$ \\ ${ }^{a}$ Graduate School of Humanities and Social Sciences, Nagoya City University, Nagoya, 467-8501, Japan, \\ yano@hum.nagoya-cu.ac.jp \\ ${ }^{b}$ Graduate School of Advanced Science and Engineering, Hiroshima University, Higashi-Hiroshima, 739-8527, Japan, \\ nisizaki@hiroshima-u.ac.jp
}

\begin{abstract}
In this paper, we propose an interactive decision making method for multiobjectve bimatrix games with fuzzy payoffs. Using possibility measure and reference membership values, an equilibrium solution concept is introduced. To circumvent computational difficulties to obtain such a solution, equilibrium conditions in the membership function space are replaced into equilibrium conditions in the expected payoff space. Under the assumption that a player can estimate the opponent player's reference membership values, the interactive algorithm is proposed to obtain a satisfactory solution of the player from among an equilibrium solution set by updating the reference membership values. A numerical example illustrates interactive processes under a hypothetical player to show the efficiency of the proposed method.
\end{abstract}

Keywords: bimatrix games with fuzzy payoff, multiobjective programming, possibility measure, an interactive method, reference membership values.

\section{Introduction}

To deal with bimatrix games with triangular fuzzy numbers, Maeda [5] defined an equilibrium solution concept using possibility measure [2], which depends on the threshold values for the level sets [2]. He formulated the corresponding mathematical programming problem to obtain such parametric equilibrium solutions. However, it is not clear how to set appropriate threshold values for the level sets. Mako et al. [6] focused on bimatrix games with LR fuzzy numbers. Corresponding to fuzzy Nash-equilibrium solution con- cept, they proposed fuzzy correlated equilibrium solution concept, which is based on a joint distribution for mixed strategies of both players. Gao [3] introduced three kinds of uncertain equilibrium solution concepts based on uncertainty theory [4], which depend on the values of confidence levels. From a similar point of view based on uncertainty theory, Tang et al. [10] proposed an uncertain equilibrium solution concept based on the Hurwicz criterion.

For multiobjective bimatrix games, Corley [1] first defined a Pareto equilibrium solution concept, and formulated quadratic programming problems to obtain Pareto equilibrium solutions through the KarushKuhn-Tucker conditions, in which multiobjective functions are scalarized by the weighting coefficients. Nishizaki et al. [8] formulated multiobjective bimatrix games incorporating fuzzy goals. They transformed multiobjective bimatrix games into usual bimatrix games by applying the weighting methods or the minimum operator [9], and defined the corresponding equilibrium solution concepts. They formulated the nonlinear programming problems to obtain such equilibrium solutions. Using dominance cones proposed by $\mathrm{Yu}$ [11], Nishizaki et al.[7] defined a nondominated equilibrium solution concept which is a generalization of Nash-equilibrium solution concept, and formulate nonlinear programming problem to obtain nondominated equilibrium solutions by applying the KarushKuhn-Tucker conditions.

In this paper, we propose an interactive decision making method for multiobjectve bimatrix games with fuzzy payoffs. Using possibility measure [2] and reference membership values [9], an equilibrium solution concept is introduced. In general, it is very difficult to directly obtain such a solution because of the computational complexity, in which each objective function based on possibility measure is expressed as a bilinear fractional function. To circumvent such computational difficulties, equilibrium conditions in the membership function space are replaced into equilibrium conditions 
in the expected payoff space. Under the assumption that a player can estimate the opponent player's reference membership values, the interactive algorithm is proposed to obtain a satisfactory solution of the player from among an equilibrium solution set by updating the reference membership values.

In section 2, we propose an interactive decision making method for multiobjectve bimatrix games. Using reference membership values, an equilibrium solution concept is defined in the membership function space. Under the asumption that a player can estimate the opponent player's reference membership values, an interactive algorithm is developed to obtain a satisfactory solution of the player from among a equilibrium solution set by updating reference membership values. In section 3 , we propose an interactive decision making method for multiobjectve bimatrix games with fuzzy payoffs. Using possibility measure and reference membership values, an equilibrium solution concept is introduced. Similar to section 2, an interactive algorithm is developed to obtain a satisfactory solution of a player from among a equilibrium solution set by updating reference membership values. In section 4, a numerical example of two-objectve bimatrix games with fuzzy payoffs illustrates interactive processes under a hypothetical player to show the efficiency of the proposed method.

\section{Interactive decision making for multiobjectve bimatrix games}

In this section, we focus on multiobjectve bimatrix games. Let $i \in\{1,2, \cdots, m\}$ be a pure strategy of Player 1 and $j \in\{1,2, \cdots, n\}$ be a pure strategy of Player 2. $A_{k}=\left(a_{k i j}\right), k=1, \ldots, K$ are Player 1 's $K$ $(m \times n)$-payoff matrices, and $B_{l}=\left(b_{l i j}\right), l=1, \cdots, L$ are Player 2's $L(m \times n)$-payoff matrices. Let $X \stackrel{\text { def }}{=}$ $\left\{\mathbf{x} \in \mathrm{R}^{m} \mid \sum_{i=1}^{m} x_{i}=1, x_{i} \geq 0, i=1, \cdots, m\right\}$ be a mixed strategy for Player 1 and let $Y \stackrel{\text { def }}{=}\left\{\mathbf{y} \in \mathrm{R}^{n} \mid \sum_{i=1}^{n} y_{j}=\right.$ $\left.1, y_{j} \geq 0, j=1, \cdots, n\right\}$ be a mixed strategy for Player 2. Then, a multiobjectve bimatrix game is formulated as follows, where $T$ means transportation.

\section{P1}

$$
\begin{aligned}
& \underset{\mathbf{x} \in X}{\operatorname{maximize}}\left(\mathbf{x}^{T} A_{1} \mathbf{y}, \cdots, \mathbf{x}^{T} A_{K}\right) \\
& \underset{\mathbf{y} \in Y}{\operatorname{maximize}}\left(\mathbf{x}^{T} B_{1} \mathbf{y}, \cdots, \mathbf{x}^{T} B_{L}\right)
\end{aligned}
$$

In this section, it is assumed that each player has fuzzy goals for multiple expected payoffs and such fuzzy goals can be defined as the following linear member- ship functions.

$$
\begin{aligned}
& \mu_{\tilde{G}_{1 k}}\left(\mathbf{x}^{T} A_{k} \mathbf{y}\right)=\frac{\mathbf{x}^{T} A_{k} \mathbf{y}-E_{k 10}}{E_{k 11}-E_{k 10}} \stackrel{\text { def }}{=} \mathbf{x}^{T} \hat{A}_{k} \mathbf{y}+c_{1 k} \\
& k=1, \cdots, K \\
& \mu_{\tilde{G}_{2 l}}\left(\mathbf{x}^{T} B_{l} \mathbf{y}\right)=\frac{\mathbf{x}^{T} B_{l} \mathbf{y}-E_{l 20}}{E_{l 21}-E_{l 20}} \stackrel{\text { def }}{=} \mathbf{x}^{T} \hat{B}_{l} \mathbf{y}+c_{2 l} \\
& l=1, \cdots, L
\end{aligned}
$$

Throughout this section, we make the following assumption.

Assumption $1 \mu_{\tilde{G}_{1 k}}\left(\mathbf{x}^{T} A_{k} \mathbf{y}\right), k=1, \cdots, K \quad$ and $\mu_{\tilde{G}_{2 l}}\left(\mathbf{x}^{T} B_{l} \mathbf{y}\right), l=1, \cdots, L$ are strictly increasing with respect to $\mathbf{x}^{T} A_{k} \mathbf{y}$ or $\mathbf{x}^{T} B_{l} \mathbf{y}$ for any $\mathbf{x} \in X, \mathbf{y} \in Y$,i.e.,

$$
\begin{gathered}
{\left[E_{k 10}, E_{k 11}\right] \supset\left\{\mathbf{x}^{T} A_{k} \mathbf{y} \in \mathrm{R}^{1} \mid \forall \mathbf{x} \in X,\right.} \\
\forall \mathbf{y} \in Y\}, k=1, \cdots, K, \\
{\left[E_{l 20}, E_{l 21}\right] \supset\left\{\mathbf{x}^{T} B_{l} \mathbf{y} \in \mathrm{R}^{1} \mid \forall \mathbf{x} \in X,\right.} \\
\forall \mathbf{y} \in Y\}, l=1, \cdots, L .
\end{gathered}
$$

When Players 1 and 2 specify reference membership values $\hat{\mu}_{1 k}, k=1, \ldots, K, \hat{\mu}_{2 l}, l=1, \ldots, L$ [9] for the above membership functions, $\mathrm{P} 1$ is reduced to a usual bimatrix game, in which the corresponding equilibrium solution is close to the corresponding reference membership values in the $L^{\infty}$ norm sense.

$\mathbf{P 2}\left(\hat{\mu}_{1}, \hat{\mu}_{2}\right)$

$$
\begin{array}{lc}
\underset{\mathbf{x} \in X}{\operatorname{maximize}} & \min _{k=1, \ldots, K}\left\{\mu_{\tilde{G}_{1 k}}\left(\mathbf{x}^{T} A_{k} \mathbf{y}\right)-\hat{\mu}_{1 k}\right\} \\
\underset{\mathbf{y} \in Y}{\operatorname{maximize}} & \min _{l=1, \ldots, L}\left\{\mu_{\tilde{G}_{2 l}}\left(\mathbf{x}^{T} B_{l} \mathbf{y}\right)-\hat{\mu}_{2 l}\right\}
\end{array}
$$

We can define the equilibrium solution to $\mathrm{P} 2\left(\hat{\mu}_{1}, \hat{\mu}_{2}\right)$, which is depend on the reference membership values $\hat{\mu}_{1 k}, k=1, \ldots, K, \hat{\mu}_{2 l}, l=1, \ldots, L$.

Definition $1\left(\mathbf{x}^{*}, \mathbf{y}^{*}\right)$ is an equilibrium solution for $P 2\left(\hat{\mu}_{1}, \hat{\mu}_{2}\right)$, if the following inequalities hold.

$$
\begin{aligned}
& \min _{k=1, \ldots, K}\left\{\mathbf{x}^{* T} \hat{A}_{k} \mathbf{y}^{*}+c_{1 k}-\hat{\mu}_{1 k}\right\} \\
\geq & \min _{k=1, \ldots, K}\left\{\mathbf{x}^{T} \hat{A}_{k} \mathbf{y}^{*}+c_{1 k}-\hat{\mu}_{1 k}\right\}, \forall \mathbf{x} \in X \\
& \min _{l=1, \ldots, L}\left\{\mathbf{x}^{* T} \hat{B}_{l} \mathbf{y}^{*}+c_{2 l}-\hat{\mu}_{2 l}\right\} \\
\geq & \min _{k=1, \ldots, K}\left\{\mathbf{x}^{* T} \hat{B}_{l} \mathbf{y}+c_{2 l}-\hat{\mu}_{2 l}\right\}, \forall \mathbf{y} \in Y
\end{aligned}
$$

Using (1), (2), P2( $\left.\hat{\mu}_{1}, \hat{\mu}_{2}\right)$ can be expressed as follows.

$$
\begin{array}{cc}
\underset{\mathbf{x} \in X}{\operatorname{maximize}} & \min _{k=1, \ldots, K}\left\{\mathbf{x}^{T} \hat{A}_{k} \mathbf{y}+c_{1 k}-\hat{\mu}_{1 k}\right\} \\
\underset{\mathbf{y} \in Y}{\operatorname{maximize}} & \min _{l=1, \ldots, L}\left\{\mathbf{x}^{T} \hat{B}_{l} \mathbf{y}+c_{2 l}-\hat{\mu}_{2 l}\right\}
\end{array}
$$


Similar to the result of Nishizaki et al. [8], an equilibrium solution to $\mathrm{P} 2\left(\hat{\mu}_{1}, \hat{\mu}_{2}\right)$ can be obtained as an optimal solution of the following nonlinear programming problem.

$$
\underset{\mathbf{x} \in X, \mathbf{y} \in Y, p, q, \sigma_{1}, \sigma_{2} \in \mathrm{R}^{1}}{\operatorname{maximize}} \sigma_{1}+\sigma_{2}-p-q
$$

subject to

$$
\begin{aligned}
& \hat{A}_{k} \mathbf{y}+\left(c_{1 k}-\hat{\mu}_{1 k}\right) \mathbf{e}_{1} \leq p \mathbf{e}_{1}, k=1, \ldots, K \\
& \mathbf{x}^{T} \hat{B}_{l}+\left(c_{2 l}-\hat{\mu}_{2 l}\right) \mathbf{e}_{2}^{T} \leq q \mathbf{e}_{2}^{T}, l=1, \ldots, L \\
& \mathbf{x}^{T} \hat{A}_{k} \mathbf{y}+\left(c_{1 k}-\hat{\mu}_{1 k}\right) \geq \sigma_{1}, k=1, \ldots, K \\
& \mathbf{x}^{T} \hat{B}_{l} \mathbf{y}+\left(c_{2 l}-\hat{\mu}_{2 l}\right) \geq \sigma_{2}, l=1, \ldots, L
\end{aligned}
$$

where $\mathbf{e}_{1}$ and $\mathbf{e}_{2}$ are $(m \times 1)$ and $(n \times 1)$ column vectors whose elements are all ones. It should be noted here that the inequalities $p \geq \sigma_{1}$ and $q \geq \sigma_{2}$ always hold in (11), because of the relationship between the constraints (11b) and (11d), or (11c) and (11e). As a result, it always holds that $\sigma_{1}+\sigma_{2}-p-q \leq 0$.

Theorem 1 Let $\left(\mathbf{x}^{*}, \mathbf{y}^{*}, p^{*}, q^{*}, \sigma_{1}^{*}, \sigma_{2}^{*}\right)$ be an optimal solution of the nonlinear programming problem (11). If $\sigma_{1}^{*}=p^{*}, \sigma_{2}^{*}=q^{*}$, then $\left(\mathbf{x}^{*}, \mathbf{y}^{*}\right)$ is an equilibrium solution to $P 2\left(\hat{\mu}_{1}, \hat{\mu}_{2}\right)$.

(Proof): Since $\left(\mathbf{x}^{*}, \mathbf{y}^{*}, p^{*}, q^{*}, \sigma_{1}^{*}, \sigma_{2}^{*}\right)$ is a feasible solution to (11), the following inequalities hold.

$$
\begin{aligned}
& \hat{A}_{k} \mathbf{y}^{*}+\left(c_{1 k}-\hat{\mu}_{1 k}\right) \mathbf{e}_{1} \leq p^{*} \mathbf{e}_{1}, k=1, \ldots, K(12) \\
& \mathbf{x}^{* T} \hat{B}_{l}+\left(c_{2 l}-\hat{\mu}_{2 l}\right) \mathbf{e}_{2}^{T} \leq q^{*} \mathbf{e}_{2}^{T}, l=1, \ldots, L(13) \\
& \mathbf{x}^{* T} \hat{A}_{k} \mathbf{y}^{*}+\left(c_{1 k}-\hat{\mu}_{1 k}\right) \geq \sigma_{1}^{*}, k=1, \ldots, K(14) \\
& \mathbf{x}^{* T} \hat{B}_{l} \mathbf{y}^{*}+\left(c_{2 l}-\hat{\mu}_{2 l}\right) \geq \sigma_{2}^{*}, l=1, \ldots, L \quad(15)
\end{aligned}
$$

From (14) and (15), it holds that

$$
\begin{aligned}
& \min _{k=1, \cdots, K}\left(\mathbf{x}^{* T} \hat{A}_{k} \mathbf{y}^{*}+\left(c_{1 k}-\hat{\mu}_{1 k}\right)\right)=\sigma_{1}^{*}, \\
& \min _{l=1, \cdots, L}\left(\mathbf{x}^{* T} \hat{B}_{l} \mathbf{y}^{*}+\left(c_{2 l}-\hat{\mu}_{2 l}\right)\right)=\sigma_{2}^{*} .
\end{aligned}
$$

On the other hand, from (12), (13), it holds that

$$
\begin{aligned}
& \max _{k=1, \cdots, K}\left(\mathbf{x}^{T} \hat{A}_{k} \mathbf{y}^{*}+\left(c_{1 k}-\hat{\mu}_{1 k}\right)\right)=p^{*} \\
& \max _{l=1, \cdots, L}\left(\mathbf{x}^{* T} \hat{B}_{l} \mathbf{y}+\left(c_{2 l}-\hat{\mu}_{2 l}\right)\right)=q^{*},
\end{aligned}
$$

for any $\mathbf{x} \in X$ and $\mathbf{y} \in Y$. As a result, from (16), (18) and $\sigma_{1}^{*}=p^{*}$, the following equilibrium condition (7) is satisfied.

$$
\begin{aligned}
& \min _{k=1, \cdots, K}\left(\mathbf{x}^{* T} \hat{A}_{k} \mathbf{y}^{*}+\left(c_{1 k}-\hat{\mu}_{1 k}\right)\right) \\
= & \max _{k=1, \cdots, K}\left(\mathbf{x}^{T} \hat{A}_{k} \mathbf{y}^{*}+\left(c_{1 k}-\hat{\mu}_{1 k}\right)\right) \\
\geq & \min _{k=1, \cdots, K}\left(\mathbf{x}^{T} \hat{A}_{k} \mathbf{y}^{*}+\left(c_{1 k}-\hat{\mu}_{1 k}\right)\right), \forall \mathbf{x} \in X
\end{aligned}
$$

In the same way, the equilibrium condition (8) is satisfied.

Under the assumption that a player can estimate the opponent player's reference membership values, the interactive algorithm is proposed to obtain a satisfactory solution of a player from among an equilibrium solution set by updating the reference membership values.

\section{Interactive algorithm 1}

Step 1 Each player sets linear membership functions $\mu_{\tilde{G}_{1 k}}(\cdot), k=1, \cdots, K, \mu_{\tilde{G}_{2 l}}(\cdot), l=1, \cdots, L$ for the fuzzy goals of expected payoffs.

Step 2 Player 1 sets the initial reference membership values $\hat{\mu}_{1 k}=1, k=1, \ldots, K$, and estimates the opponent player's reference membership values $\hat{\mu}_{2 l}, l=1, \ldots, L$.

Step 3 Solve the nonlinear programming problem (11), and obtain the equilibrium solution $\left(\mathbf{x}^{*}, \mathbf{y}^{*}\right)$ to $\mathrm{P} 2\left(\hat{\mu}_{1}, \hat{\mu}_{2}\right)$.

Step 4 If Player 1 is satisfied with the current values of the membership functions $\mu_{\tilde{G}_{1 k}}\left(\mathbf{x}^{* T} \hat{A}_{k} \mathbf{y}^{*}\right), k=$ $1, \ldots, K$, then stop. Otherwise, update the reference membership values $\hat{\mu}_{1 k}, k=1, \ldots, K$, and go to Step 3.

\section{Interactive decision making for multiobjective bimatrix games with fuzzy payoffs}

\subsection{Multiobjective bimatrix games with fuzzy payoffs}

In this section, we consider multiobjective bimatrix games with fuzzy payoffs. Let $i \in\{1,2, \cdots, m\}$ be a pure strategy of Player 1 and $j \in\{1,2, \cdots, n\}$ be a pure strategy of Player 2. $\tilde{A}_{k}=\left(\tilde{a}_{k i j}\right), k=1, \ldots, K$ are Player 1's $K(m \times n)$-payoff matrices, and $\tilde{B}_{l}=$ $\left(\tilde{b}_{l i j}\right), l=1, \cdots, L$ are Player 2's $L(m \times n)$-payoff matrices. Elements $\tilde{a}_{k i j}$ and $\tilde{b}_{l i j}$ are LR fuzzy numbers [2] whose membership functions are defined as follows.

$$
\begin{gathered}
\mu_{\widetilde{a}_{k i j}}(s)= \begin{cases}L_{1}\left(\frac{a_{k i j}-s}{\alpha_{k i j}}\right) & a_{k i j}-\alpha_{k i j} \leq s \leq a_{k i j} \\
R_{1}\left(\frac{s-a_{k i j}}{\beta_{k i j}}\right) & a_{k i j} \leq s \leq a_{k i j}+\beta_{k i j}\end{cases} \\
\mu_{\widetilde{b}_{l i j}}(t)= \begin{cases}L_{2}\left(\frac{b_{l i j}-t}{\gamma_{l i j}}\right) & b_{l i j}-\gamma_{l i j} \leq t \leq b_{l i j} \\
R_{2}\left(\frac{t-b_{l i j}}{\delta_{l i j}}\right) & b_{l i j} \leq t \leq b_{l i j}+\delta_{l i j}\end{cases}
\end{gathered}
$$

where $L_{1}(\cdot)$ is a type function [2] which is strictly monotone decreasing on the interval $[0,1]$, and $L_{1}(0)=1, L_{1}(1)=0$. For the other type functions $R_{1}(\cdot), L_{2}(\cdot), R_{2}(\cdot)$, similar conditions are satisfied. Let 
$X \stackrel{\text { def }}{=}\left\{\mathbf{x} \in \mathrm{R}^{m} \mid \sum_{i=1}^{m} x_{i}=1, x_{i} \geq 0, i=1, \cdots, m\right\}$ be a mixed strategy for Player 1 and let $Y \stackrel{\text { def }}{=}\left\{\mathbf{y} \in \mathrm{R}^{n} \mid\right.$ $\left.\sum_{i=1}^{n} y_{j}=1, y_{j} \geq 0, j=1, \cdots, n\right\}$ be a mixed strategy for Player 2. Then, a multiobjectve bimatrix game with fuzzy payoffs is formally expressed as follows.

P3

$$
\begin{aligned}
& \underset{\mathbf{x} \in X}{\operatorname{maximize}}\left(\mathbf{x}^{T} \tilde{A}_{1} \mathbf{y}, \ldots, \mathbf{x}^{T} \tilde{A}_{K} \mathbf{y}\right) \\
& \underset{\mathbf{y} \in Y}{\operatorname{maximize}}\left(\mathbf{x}^{T} \tilde{B}_{1} \mathbf{y}, \ldots, \mathbf{x}^{T} \tilde{B}_{L} \mathbf{y}\right)
\end{aligned}
$$

In P3, it should be noted here that fuzzy expected payoffs $\mathbf{x}^{T} \tilde{A}_{k} \mathbf{y}, k=1, \cdots, K$ and $\mathbf{x}^{T} \tilde{B}_{l} \mathbf{y}, l=1, \cdots, L$ are expressed as LR fuzzy numbers because of the property [2] of LR fuzzy numbers. We assume that Players 1 and 2 have fuzzy goals $\tilde{G}_{1 k}, k=1, \cdots, K, \tilde{G}_{2 l}, l=$ $1, \cdots, L$ for their expected payoffs, and the corresponding membership functions are linear ones defined as follows.

$$
\begin{gathered}
\mu_{\tilde{G}_{1 k}}(s)=\frac{s-E_{k 10}}{E_{k 11}-E_{k 10}}, k=1, \ldots, K \\
\mu_{\tilde{G}_{2 l}}(t)=\frac{t-E_{l 20}}{E_{l 21}-E_{l 20}}, l=1, \ldots, L
\end{gathered}
$$

Throughout this section, we make the following assumption.

Assumption $2 \mu_{\tilde{G}_{1 k}}(s), k=1, \cdots, K, \quad \mu_{\tilde{G}_{2 l}}(t), l=$ $1, \cdots, L$ are strictly increasing on the corresponding support for LR fuzzy numbers $\mathbf{x}^{T} \tilde{A}_{k} \mathbf{y}$ or $\mathbf{x}^{T} \tilde{B}_{l} \mathbf{y}$, i.e.,

$$
\begin{array}{r}
{\left[E_{k 10}, E_{k 11}\right] \supset\left\{s \in R^{1} \mid \mu_{\mathbf{x}^{T} \tilde{A}_{k} \mathbf{y}}(s)>0,\right.} \\
\forall \mathbf{x} \in X, \forall \mathbf{y} \in Y\}, k=1, \cdots, K, \\
{\left[E_{l 20}, E_{l 21}\right] \supset\left\{t \in R^{1} \mid \mu_{\mathbf{x}^{T} \tilde{B}_{l} \mathbf{y}}(t)>0,\right.} \\
\forall \mathbf{x} \in X, \forall \mathbf{y} \in Y\}, l=1, \cdots, L .
\end{array}
$$

By introducing possibility measure [2], P3 can be interpreted as the following problem.

\section{P4}

$$
\begin{gathered}
\underset{\mathbf{x} \in X}{\operatorname{maximize}}\left(\Pi_{\mathbf{x}^{T} \tilde{A}_{1} \mathbf{y}}\left(\tilde{G}_{11}\right), \ldots, \Pi_{\mathbf{x}^{T} \tilde{A}_{K} \mathbf{y}}\left(\tilde{G}_{1 K}\right)\right) \\
\underset{\mathbf{y} \in Y}{\operatorname{maximize}}\left(\Pi_{\mathbf{x}^{T} \tilde{B}_{1 \mathbf{y}}}\left(\tilde{G}_{21}\right), \ldots, \Pi_{\mathbf{x}^{T} \tilde{B}_{L} \mathbf{y}}\left(\tilde{G}_{2 L}\right)\right),
\end{gathered}
$$

where the objective functions of $\mathrm{P} 4$ are expressed as the bilinear fractional functions.

$$
\begin{aligned}
& \Pi_{\mathbf{x}^{T} \tilde{A}_{k} \mathbf{y}}\left(\tilde{G}_{1 k}\right)=\frac{\sum_{i=1}^{m} \sum_{j=1}^{n}\left(a_{k i j}+\beta_{k i j}\right) x_{i} y_{j}-E_{k 10}}{E_{k 11}-E_{k 10}+\sum_{i=1}^{m} \sum_{j=1}^{n} \beta_{k i j} x_{i} y_{j}}, \\
& k=1, \ldots, K \\
& \Pi_{\mathbf{x}^{T} \tilde{B}_{l} \mathbf{y}}\left(\tilde{G}_{2 l}\right)=\frac{\sum_{i=1}^{m} \sum_{j=1}^{n}\left(b_{l i j}+\delta_{l i j}\right) x_{i} y_{j}-E_{l 20}}{E_{l 21}-E_{l 20}+\sum_{i=1}^{m} \sum_{j=1}^{n} \delta_{l i j} x_{i} y_{j}}, \\
& l=1, \ldots, L
\end{aligned}
$$

From the assumption for the parameters $E_{k 10}, E_{k 11}$, $E_{l 20}, E_{l 21}$, the following relations always hold.

$$
\begin{aligned}
& 0<\Pi_{\mathbf{x}^{T} \tilde{A}_{k} \mathbf{y}}\left(\tilde{G}_{1 k}\right)<1, k=1, \ldots, K, \forall \mathbf{x} \in X, \forall \mathbf{y} \in Y \\
& 0<\Pi_{\mathbf{x}^{T} \tilde{B}_{l} \mathbf{y}}\left(\tilde{G}_{2 l}\right)<1, l=1, \ldots, L, \forall \mathbf{x} \in X, \forall \mathbf{y} \in Y
\end{aligned}
$$

To define an equilibrium solution concept to $\mathrm{P} 4$, We assume that Players 1 and 2 can specify the reference membership values $\hat{\mu}_{1 k}, k=1, \ldots, K$ or $\hat{\mu}_{2 l}, l=$ $1, \ldots, L$. Then, $\mathrm{P} 4$ can be reduced to the following bimatrix game.

$$
\begin{gathered}
\mathbf{P 5}\left(\hat{\mu}_{1}, \hat{\mu}_{2}\right) \\
\underset{\mathbf{x} \in X}{\operatorname{maximize}} \min _{k=1, \ldots, K}\left\{\Pi_{\mathbf{x}^{T} \tilde{A}_{k} \mathbf{y}}\left(\tilde{G}_{1 k}\right)-\hat{\mu}_{1 k}\right\} \\
\underset{\mathbf{y} \in Y}{\operatorname{maximize}} \min _{l=1, \ldots, L}\left\{\Pi_{\mathbf{x}^{T} \tilde{B}_{l} \mathbf{y}}\left(\tilde{G}_{2 l}\right)-\hat{\mu}_{2 l}\right\}
\end{gathered}
$$

Now, we can introduce an equilibrium solution concept for P5 $\left(\hat{\mu}_{1}, \hat{\mu}_{2}\right)$, which depends on the reference membership values $\hat{\mu}_{1 k}, k=1, \ldots, K, \hat{\mu}_{2 l}, l=1, \ldots, L$.

Definition $2\left(\mathbf{x}^{*}, \mathbf{y}^{*}\right)$ is an equilibrium solution to $P 5\left(\hat{\mu}_{1}, \hat{\mu}_{2}\right)$,if the following inequalities hold.

$$
\begin{aligned}
& \min _{k=1, \ldots, K}\left\{\Pi_{\mathbf{x}^{* T} \tilde{A}_{k} \mathbf{y}^{*}}\left(\tilde{G}_{1 k}\right)-\hat{\mu}_{1 k}\right\} \\
& \geq \min _{k=1, \ldots, K}\left\{\Pi_{\mathbf{x}^{T} \tilde{A}_{k} \mathbf{y}^{*}}\left(\tilde{G}_{1 k}\right)-\hat{\mu}_{1 k}\right\}, \forall \mathbf{x} \in X \\
& \min _{l=1, \ldots, L}\left\{\Pi_{\mathbf{x}^{* T} \tilde{B}_{l} \mathbf{y}^{*}}\left(\tilde{G}_{2 l}\right)-\hat{\mu}_{2 l}\right\} \\
& \geq \min _{l=1, \ldots, L}\left\{\Pi_{\mathbf{x}^{* T} \tilde{B}_{l} \mathbf{y}}\left(\tilde{G}_{2 l}\right)-\hat{\mu}_{2 l}\right\}, \forall \mathbf{y} \in Y
\end{aligned}
$$

It is very difficult to obtain the equilibrium solution to P5 $\left(\hat{\mu}_{1}, \hat{\mu}_{2}\right)$ in the computational aspect, since (26) and (27) are bilinear fractional functions. To circumvent such a difficulty, at first, we consider the following bimatrix game, which is equivalent to $\mathrm{P} 5\left(\hat{\mu}_{1}, \hat{\mu}_{2}\right)$.

P6 $\left(\hat{\mu}_{1}, \hat{\mu}_{2}\right)$

$$
\begin{aligned}
& \underset{\mathbf{x} \in X, v_{1} \in \mathrm{R}^{1}}{\operatorname{maximize}} v_{1} \\
& \text { subject to } \\
& \Pi_{\mathbf{x}^{T} \tilde{A}_{k} \mathbf{y}}\left(\tilde{G}_{1 k}\right)-\hat{\mu}_{1 k} \geq v_{1}, k=1, \ldots, K \\
& \operatorname{maximize} v_{2} \\
& \mathbf{y} \in Y, v_{2} \in \mathrm{R}^{1} \\
& \text { subject to } \\
& \Pi_{\mathbf{x}^{T} \tilde{B}_{l} \mathbf{y}}\left(\tilde{G}_{2 l}\right)-\hat{\mu}_{2 l} \geq v_{2}, l=1, \ldots, L
\end{aligned}
$$

Let $\left(\mathbf{x}^{*}, \mathbf{y}^{*}, v_{1}^{*}, v_{2}^{*}\right)$ be an equilibrium solution to P6 $\left(\hat{\mu}_{1}, \hat{\mu}_{2}\right)$. Then, the following equalities hold at 


$$
\begin{aligned}
& \left(\mathbf{x}^{*}, \mathbf{y}^{*}, v_{1}^{*}, v_{2}^{*}\right) . \\
& \min _{k=1, \ldots, K}\left(\Pi_{\mathbf{x}^{* T} \tilde{A}_{k} \mathbf{y}^{*}}\left(\tilde{G}_{1 k}\right)-\left(v_{1}^{*}+\hat{\mu}_{1 k}\right)\right)=0 \\
& \min _{l=1, \ldots, L}\left(\Pi_{\mathbf{x}^{* T} \tilde{B}_{l} \mathbf{y}^{*}}\left(\tilde{G}_{2 l}\right)-\left(v_{2}^{*}+\hat{\mu}_{2 l}\right)\right)=0
\end{aligned}
$$

We denote $\left(v_{1}^{*}+\hat{\mu}_{1 k}\right)$-level sets for LR fuzzy numbers $\mathbf{x}^{* T} \tilde{A}_{k} \mathbf{y}^{*}$ and $\mathbf{x}^{* T} \tilde{B}_{l} \mathbf{y}^{*}$ as the closed intervals $\quad\left[\mathbf{x}^{* T} A_{k, v_{1}^{*}+\hat{\mu}_{1 k}}^{L} \mathbf{y}^{*}, \mathbf{x}^{* T} A_{k, v_{1}^{*}+\hat{\mu}_{1 k}}^{R} \mathbf{y}^{*}\right] \quad$ and $\left[\mathbf{x}^{* T} B_{l, v_{2}^{*}+\hat{\mu}_{2 l}}^{L} \mathbf{y}^{*}, \mathbf{x}^{* T} B_{l, v_{2}^{*}+\hat{\mu}_{2 l}}^{R} \mathbf{y}^{*}\right]$, respectively, where

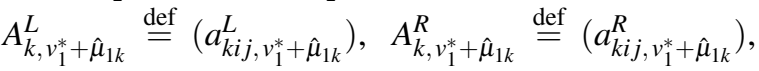

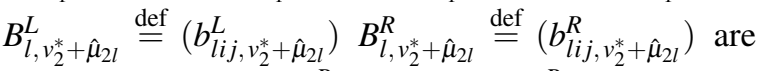
$(m \times n)$-matrices. $\quad a_{k i j, v_{1}^{*}+\hat{\mu}_{1 k}}$ and $b_{l i j, v_{2}^{*}+\hat{\mu}_{2 l}}$ mean the right side extreme point of the $\left(v_{1}^{*}+\hat{\mu}_{1 k}\right)$-level set for $\tilde{a}_{k i j}$, and the right side extreme point of the $\left(v_{2}^{*}+\hat{\mu}_{2 l}\right)$-level set for $\tilde{b}_{l i j}$.

It is obvious that (33a) and (33b) are equivalent to the following equalities, respectively, because of (28) and (29).

$$
\begin{aligned}
& \min _{k=1, \ldots, K}\left(\mathbf{x}^{* T} A_{k, v_{1}^{*}+\hat{\mu}_{1 k}}^{R} \mathbf{y}^{*}-\mu_{\tilde{G}_{1 k}}^{-1}\left(v_{1}^{*}+\hat{\mu}_{1 k}\right)\right)=0 \\
& \min _{l=1, \ldots, L}\left(\mathbf{x}^{* T} B_{l, v_{2}^{*}+\hat{\mu}_{2 l}}^{R} \mathbf{y}^{*}-\mu_{\tilde{G}_{2 l}}^{-1}\left(v_{2}^{*}+\hat{\mu}_{2 l}\right)\right)=0
\end{aligned}
$$

Corresponding to (34a) and (34b), we consider the following bimatrix game, in which $\left(\hat{\mu}_{1}, \hat{\mu}_{2}\right)$ and $\left(v_{1}^{*}, v_{2}^{*}\right)$ are given in advance.

$$
\begin{aligned}
& \mathbf{P 7}\left(\hat{\mu}_{1}, \hat{\mu}_{2} ; v_{1}^{*}, v_{2}^{*}\right) \\
& \underset{\mathbf{x} \in X}{\operatorname{maximize}} \min _{k=1, \ldots, K}\left\{\mathbf{x}^{T} A_{k, v_{1}^{*}+\hat{\mu}_{1 k}}^{R} \mathbf{y}-\mu_{\tilde{G}_{1 k}}^{-1}\left(v_{1}^{*}+\hat{\mu}_{1 k}\right)\right\} \\
& \underset{\mathbf{y} \in Y}{\operatorname{maximize}} \min _{l=1, \ldots, L}\left\{\mathbf{x}^{T} B_{l, v_{2}^{*}+\hat{\mu}_{2 l}}^{R} \mathbf{y}-\mu_{\tilde{G}_{2 l}}^{-1}\left(v_{2}^{*}+\hat{\mu}_{2 l}\right)\right\}
\end{aligned}
$$

For P7 $\left(\hat{\mu}_{1}, \hat{\mu}_{2} ; v_{1}^{*}, v_{2}^{*}\right)$, we introduce an equilibrium solution concept.

Definition $3\left(\mathbf{x}^{*}, \mathbf{y}^{*}\right)$ is an equilibrium solution for $P 7\left(\hat{\mu}_{1}, \hat{\mu}_{2} ; v_{1}^{*}, v_{2}^{*}\right)$, if the following inequalities hold.

$$
\begin{aligned}
& \min _{k=1, \ldots, K}\left\{\mathbf{x}^{* T} A_{k, v_{1}^{*}+\hat{\mu}_{1 k}}^{R} \mathbf{y}^{*}-\mu_{\tilde{G}_{1 k}}^{-1}\left(v_{1}^{*}+\hat{\mu}_{1 k}\right)\right\} \\
& \geq \min _{k=1, \ldots, K}\left\{\mathbf{x}^{T} A_{k, v_{1}^{*}+\hat{\mu}_{1 k}} \mathbf{y}^{*}-\mu_{\tilde{G}_{1 k}}^{-1}\left(v_{1}^{*}+\hat{\mu}_{1 k}\right)\right\}, \forall \mathbf{x} \in X \\
& \min _{l=1, \ldots, L}\left\{\mathbf{x}^{* T} B_{l, v_{2}^{*}+\hat{\mu}_{2 l}} \mathbf{y}^{*}-\mu_{\tilde{G}_{2 l}}^{-1}\left(v_{2}^{*}+\hat{\mu}_{2 l}\right)\right\} \\
& \geq \min _{l=1, \ldots, L}\left\{\mathbf{x}^{* T} B_{l, v_{2}^{*}+\hat{\mu}_{2 l}}^{R} \mathbf{y}-\mu_{\tilde{G}_{2 l}}^{-1}\left(v_{2}^{*}+\hat{\mu}_{2 l}\right)\right\}, \forall \mathbf{y} \in Y
\end{aligned}
$$

Then, the following relationships between equilibrium solutions for $\mathrm{P} 7\left(\hat{\mu}_{1}, \hat{\mu}_{2} ; v_{1}^{*}, v_{2}^{*}\right)$ and equilibrium solutions to $\mathrm{P} 6\left(\hat{\mu}_{1}, \hat{\mu}_{2}\right)$ hold.
Theorem 2 If $\left(\mathbf{x}^{*}, \mathbf{y}^{*}, v_{1}^{*}, v_{2}^{*}\right)$ is an equilibrium solution to P6 $\left(\hat{\mu}_{1}, \hat{\mu}_{2}\right)$, then $\left(\mathbf{x}^{*}, \mathbf{y}^{*}\right)$ is an equilibrium solution for $P 7\left(\hat{\mu}_{1}, \hat{\mu}_{2} ; v_{1}^{*}, v_{2}^{*}\right)$.

(Proof) : Assume that $\left(\mathbf{x}^{*}, \mathbf{y}^{*}\right)$ is not an equilibrium solution for $\mathrm{P} 7\left(\hat{\mu}_{1}, \hat{\mu}_{2} ; v_{1}^{*}, v_{2}^{*}\right)$. Then, there exists some $\mathbf{x} \in X$ such that

$$
\begin{aligned}
& \min _{k=1, \ldots, K}\left\{\mathbf{x}^{* T} A_{k, v_{1}^{*}+\hat{\mu}_{1 k}}^{R} \mathbf{y}^{*}-\mu_{\tilde{G}_{1 k}}^{-1}\left(v_{1}^{*}+\hat{\mu}_{1 k}\right)\right\}< \\
& \min _{k=1, \ldots, K}\left\{\mathbf{x}^{T} A_{k, v_{1}^{*}+\hat{\mu}_{1 k}}^{R} \mathbf{y}^{*}-\mu_{\tilde{G}_{1 k}}^{-1}\left(v_{1}^{*}+\hat{\mu}_{1 k}\right)\right\},(37)
\end{aligned}
$$

or, there exists some $\mathbf{y} \in Y$ such that

$$
\begin{aligned}
& \min _{l=1, \ldots, L}\left\{\mathbf{x}^{* T} B_{l, v_{2}^{*}+\hat{\mu}_{2 l}}^{R} \mathbf{y}^{*}-\mu_{\tilde{G}_{2 l}}^{-1}\left(v_{2}^{*}+\hat{\mu}_{2 l}\right)\right\}< \\
& \min _{l=1, \ldots, L}\left\{\mathbf{x}^{* T} B_{l, v_{2}^{*}+\hat{\mu}_{2 l}}^{R} \mathbf{y}-\mu_{\tilde{G}_{2 l}}^{-1}\left(v_{2}^{*}+\hat{\mu}_{2 l}\right)\right\} .
\end{aligned}
$$

Assume that there exists some $\mathbf{x} \in X$ such that the inequality (37) is satisfied. Then, from (34a), the following relation holds.

$$
\begin{aligned}
0 & =\min _{k=1, \cdots, K}\left(\mathbf{x}^{* T} A_{k, v_{1}^{*}+\hat{\mu}_{1 k}}^{R} \mathbf{y}^{*}-\mu_{\tilde{G}_{1 k}}^{-1}\left(v_{1}^{*}+\hat{\mu}_{1 k}\right)\right) \\
& <\min _{k=1, \cdots, K}\left(\mathbf{x}^{T} A_{k, v_{1}^{*}+\hat{\mu}_{1 k}}^{R} \mathbf{y}^{*}-\mu_{\tilde{G}_{1 k}}^{-1}\left(v_{1}^{*}+\hat{\mu}_{1 k}\right)\right)
\end{aligned}
$$

Since $\mu_{\tilde{G}_{1 k}}(\cdot)$ is strictly monotone increasing and the right hand side function of the membership function of $\mathbf{x}^{T} \tilde{A} \mathbf{y}^{*}$ is strictly monotone decreasing, the above relation is equivalent to the following inequality.

$$
\begin{aligned}
v_{1}^{*} & =\min _{k=1, \cdots, K}\left(\Pi_{\mathbf{x}^{* T} \tilde{A}_{k} \mathbf{y}^{*}}\left(\tilde{G}_{1 k}\right)-\hat{\mu}_{1 k}\right) \\
& <\min _{k=1, \cdots, K}\left(\Pi_{\mathbf{x}^{T} \tilde{A}_{k} \mathbf{y}^{*}}\left(\tilde{G}_{1 k}\right)-\hat{\mu}_{1 k}\right) .
\end{aligned}
$$

This contradicts the fact that $\left(\mathbf{x}^{*}, \mathbf{y}^{*}, v_{1}^{*}, v_{2}^{*}\right)$ is an equilibrium solution to $\mathrm{P} 6\left(\hat{\mu}_{1}, \hat{\mu}_{2}\right)$. Similarly, we can prove for the case that there exists $\mathbf{y} \in Y$ such that (38) is satisfied.

Theorem 3 If $\left(\mathbf{x}^{*}, \mathbf{y}^{*}\right)$ is an equilibrium solution to $P 7\left(\hat{\mu}_{1}, \hat{\mu}_{2} ; v_{1}^{*}, v_{2}^{*}\right)$, where the following relations hold,

$$
\begin{aligned}
& \min _{k=1, \cdots, K}\left(\mathbf{x}^{* T} A_{k, v_{1}^{*}+\hat{\mu}_{1 k}}^{R} \mathbf{y}^{*}-\mu_{\tilde{G}_{1 k}}^{-1}\left(v_{1}^{*}+\hat{\mu}_{1 k}\right)\right)=0 \\
& \min _{l=1, \cdots, L}\left(\mathbf{x}^{* T} B_{l, v_{2}^{*}+\hat{\mu}_{2 l}}^{R} \mathbf{y}^{*}-\mu_{\tilde{G}_{2 l}}^{-1}\left(v_{2}^{*}+\hat{\mu}_{2 l}\right)\right)=0,
\end{aligned}
$$

then, $\left(\mathbf{x}^{*}, \mathbf{y}^{*}, v_{1}^{*}, v_{2}^{*}\right)$ is an equilibrium solution to $P 6\left(\hat{\mu}_{1}, \hat{\mu}_{2}\right)$.

(Proof) : Assume that $\left(\mathbf{x}^{*}, \mathbf{y}^{*}, v_{1}^{*}, v_{2}^{*}\right)$ is not an equilibrium solution to $\mathrm{P} 6\left(\hat{\mu}_{1}, \hat{\mu}_{2}\right)$. Then, there exists some $\mathbf{x} \in X$ such that

$$
\begin{array}{r}
\min _{k=1, \ldots, K}\left\{\Pi_{\mathbf{x}^{* T} \tilde{A}_{k} \mathbf{y}^{*}}\left(\tilde{G}_{1 k}\right)-\hat{\mu}_{1 k}\right\} \\
<\min _{k=1, \ldots, K}\left\{\Pi_{\mathbf{x}^{T} \tilde{A}_{k} \mathbf{y}^{*}}\left(\tilde{G}_{1 k}\right)-\hat{\mu}_{1 k}\right\},
\end{array}
$$


or, there exists some $\mathbf{y} \in Y$ such that

$$
\begin{aligned}
& \min _{l=1, \ldots, L}\left\{\Pi_{\mathbf{x}^{* T} \tilde{B}_{l} \mathbf{y}^{*}}\left(\tilde{G}_{2 l}\right)-\hat{\mu}_{2 l}\right\} \\
< & \min _{l=1, \ldots, L}\left\{\Pi_{\mathbf{x}^{* T} \tilde{B}_{l} \mathbf{y}}\left(\tilde{G}_{2 l}\right)-\hat{\mu}_{2 l}\right\} .
\end{aligned}
$$

Assume that there exists some $\mathbf{x} \in X$ such that (41) is satisfied. Then, from (39), it holds that

$$
\begin{aligned}
v_{1}^{*} & =\min _{k=1, \ldots, K}\left(\Pi_{\mathbf{x}^{* T} \tilde{A}_{k} \mathbf{y}^{*}}\left(\tilde{G}_{1 k}\right)-\hat{\mu}_{1 k}\right) \\
& <\min _{k=1, \ldots, K}\left(\Pi_{\mathbf{x}^{T} \tilde{A}_{k} \mathbf{y}^{*}}\left(\tilde{G}_{1 k}\right)-\hat{\mu}_{1 k}\right) .
\end{aligned}
$$

This means that the following relation holds.

$$
\begin{aligned}
& 0=\min _{k=1, \cdots, K}\left(\mathbf{x}^{* T} A_{k, v_{1}^{*}+\hat{\mu}_{1 k}} \mathbf{y}^{*}-\mu_{\tilde{G}_{1 k}}^{-1}\left(v_{1}^{*}+\hat{\mu}_{1 k}\right)\right) \\
& <\min _{k=1, \cdots, K}\left(\mathbf{x}^{T} A_{k, v_{1}^{*}+\hat{\mu}_{1 k}}^{R} \mathbf{y}^{*}-\mu_{\tilde{G}_{1 k}}^{-1}\left(v_{1}^{*}+\hat{\mu}_{1 k}\right)\right)
\end{aligned}
$$

This contradicts the fact that $\left(\mathbf{x}^{*}, \mathbf{y}^{*}\right)$ is an equilibrium solution to $\mathrm{P} 7\left(\hat{\mu}_{1}, \hat{\mu}_{2} ; v_{1}^{*}, v_{2}^{*}\right)$. Similarly, we can prove for the case that there exists $\mathbf{y} \in Y$ such that (42) is satisfied.

From the above theorems, instead of solving P6 $\left(\hat{\mu}_{1}, \hat{\mu}_{2}\right)$ directly, we can obtain an equilibrium solution to $\mathrm{P} 6\left(\hat{\mu}_{1}, \hat{\mu}_{2}\right)$ by solving $\mathrm{P} 7\left(\hat{\mu}_{1}, \hat{\mu}_{2} ; v_{1}^{*}, v_{2}^{*}\right)$, where $\left(v_{1}^{*}, v_{2}^{*}\right)$ satisfies the equality conditions (39) and (40). On the other hand, an equilibrium solution to P7 $\left(\hat{\mu}_{1}, \hat{\mu}_{2} ; v_{1}^{*}, v_{2}^{*}\right)$ is obtained by solving the following nonlinear programming problem [8].

$\mathbf{P 8}\left(\hat{\mu}_{1}, \hat{\mu}_{2} ; v_{1}^{*}, v_{2}^{*}\right)$

$$
\underset{\mathbf{x} \in X, \mathbf{y} \in Y, p, q, \sigma_{1}, \sigma_{2}}{\operatorname{maximize}} \sigma_{1}+\sigma_{2}-p-q
$$

subject to

$$
A_{k, v_{1}^{*}+\hat{\mu}_{1 k}}^{R} \mathbf{y}-\mu_{\tilde{G}_{1 k}}^{-1}\left(v_{1}^{*}+\hat{\mu}_{1 k}\right) \mathbf{e}_{1} \leq p \mathbf{e}_{1}, k=1, \ldots, K
$$

$$
\mathbf{x}^{T} B_{l, v_{2}^{*}+\hat{\mu}_{2 l}}^{R}-\mu_{\tilde{G}_{2 l}}^{-1}\left(v_{2}^{*}+\hat{\mu}_{2 l}\right) \mathbf{e}_{2} \leq q \mathbf{e}_{2}, l=1, \ldots, L
$$

$$
\begin{aligned}
& \mathbf{x}^{T} A_{k, v_{1}^{*}+\hat{\mu}_{1 k}}^{R} \mathbf{y}-\mu_{\tilde{G}_{1 k}}^{-1}\left(v_{1}^{*}+\hat{\mu}_{1 k}\right) \geq \sigma_{1}, k=1, \ldots, K \\
& \mathbf{x}^{T} B_{l, v_{2}^{*}+\hat{\mu}_{2 l}}^{R} \mathbf{y}-\mu_{\tilde{G}_{2 l}}^{-1}\left(v_{2}^{*}+\hat{\mu}_{2 l}\right) \geq \sigma_{2}, l=1, \ldots, L,
\end{aligned}
$$

where $\mathbf{e}_{1}$ and $\mathbf{e}_{2}$ are $(m \times 1)$ and $(n \times 1)$ column vectors whose elements are all ones. Similar to the nonlinear programming problem (11), $p \geq \sigma_{1}, q \geq \sigma_{2}$ and in $\mathrm{P} 8\left(\hat{\mu}_{1}, \hat{\mu}_{2} ; v_{1}^{*}, v_{2}^{*}\right), \sigma_{1}+\sigma_{2}-p-q \leq 0$ always holds.

Similar to Theorem 1, the following theorem shows the relationship between an optimal solution to $\mathrm{P} 8\left(\hat{\mu}_{1}, \hat{\mu}_{2} ; v_{1}^{*}, v_{2}^{*}\right)$ and an equilibrium solution to $\mathrm{P} 6\left(\hat{\mu}_{1}, \hat{\mu}_{2}\right)$.
Theorem 4 Let $\left(\mathbf{x}^{*}, \mathbf{y}^{*}, p^{*}, q^{*}, \sigma_{1}^{*}, \sigma_{2}^{*}\right)$ be an optimal solution to $P 8\left(\hat{\mu}_{1}, \hat{\mu}_{2} ; v_{1}^{*}, v_{2}^{*}\right)$. If $\sigma_{1}^{*}=p^{*}=0, \sigma_{2}^{*}=$ $q^{*}=0$, then $\left(\mathbf{x}^{*}, \mathbf{y}^{*}\right)$ is an equilibrium solution for $P 6\left(\hat{\mu}_{1}, \hat{\mu}_{2}\right)$.

(Proof): The equality conditions (39),(40) in Theorem 3 are satisfied because of $\sigma_{1}^{*}=\sigma_{2}^{*}=0$. According to $\sigma_{1}^{*}=p^{*}, \sigma_{2}^{*}=q^{*}$ and Theorem 1 , an optimal solution $\left(\mathbf{x}^{*}, \mathbf{y}^{*}, p^{*}, q^{*}, \sigma_{1}^{*}, \sigma_{2}^{*}\right)$ to $\mathrm{P} 8\left(\hat{\mu}_{1}, \hat{\mu}_{2} ; v_{1}^{*}, v_{2}^{*}\right)$ satisfies the equilibrium conditions to $\mathrm{P} 7\left(\hat{\mu}_{1}, \hat{\mu}_{2} ; v_{1}^{*}, v_{2}^{*}\right)$. From Definition 3 , the following inequalities hold.

$$
\begin{aligned}
& 0=\min _{k=1, \cdots, K}\left(\mathbf{x}^{*} A_{k, v_{1}+\hat{\mu}_{1 k}}^{R} \mathbf{y}^{*}-\mu_{\tilde{G}_{1 k}}^{-1}\left(v_{1}^{*}+\hat{\mu}_{1 k}\right)\right) \\
& \geq \min _{k=1, \ldots, K}\left(\mathbf{x}^{T} A_{k, v_{1}^{*}+\hat{\mu}_{1 k}}^{R} \mathbf{y}^{*}-\mu_{\tilde{G}_{1 k}}^{-1}\left(v_{1}^{*}+\hat{\mu}_{1 k}\right)\right), \\
& \forall x \in X \\
& 0=\min _{l=1, \cdots, L}\left(\mathbf{x}^{*} B_{l, v_{2}+\hat{\mu}_{2 l}}^{R} \mathbf{y}^{*}-\mu_{\tilde{G}_{2 l}}^{-1}\left(v_{2}^{*}+\hat{\mu}_{2 l}\right)\right) \\
& \geq \min _{l=1, \ldots, L}\left(\mathbf{x}^{* T} B_{l, v_{2}^{*}+\hat{\mu}_{2 l}}^{R} \mathbf{y}-\mu_{\tilde{G}_{2 l}}^{-1}\left(v_{2}^{*}+\hat{\mu}_{2 l}\right)\right), \\
& \forall y \in Y
\end{aligned}
$$

The above inequalities can be equivalently expressed as follows.

$$
\begin{aligned}
& v_{1}^{*}=\min _{k=1, \ldots, K}\left\{\Pi_{\mathbf{x}^{* T} \tilde{A}_{k} \mathbf{y}^{*}}\left(\tilde{G}_{1 k}\right)-\hat{\mu}_{1 k}\right\} \\
& \geq \min _{k=1, \ldots, K}\left\{\Pi_{\mathbf{x}^{T} \tilde{A}_{k} \mathbf{y}^{*}}\left(\tilde{G}_{1 k}\right)-\hat{\mu}_{1 k}\right\}, \forall x \in X \\
& v_{2}^{*}=\min _{l=1, \ldots, L}\left\{\Pi_{\mathbf{x}^{* T} \tilde{B}_{l} \mathbf{y}^{*}}\left(\tilde{G}_{2 l}\right)-\hat{\mu}_{2 l}\right\} \\
& \geq \min _{l=1, \ldots, L}\left\{\Pi_{\mathbf{x}^{* T} \tilde{B}_{l} \mathbf{y}}\left(\tilde{G}_{2 l}\right)-\hat{\mu}_{2 l}\right\}, \forall y \in Y
\end{aligned}
$$

This means that an optimal solution to $\mathrm{P} 8\left(\hat{\mu}_{1}, \hat{\mu}_{2} ; v_{1}^{*}, v_{2}^{*}\right)$ is an equilibrium solution to $\operatorname{P6}\left(\hat{\mu}_{1}, \hat{\mu}_{2}\right)$.

The relationships between an equilibrium solution to $\mathrm{P} 6\left(\hat{\mu}_{1}, \hat{\mu}_{2}\right)$, an equilibrium solution to $\mathrm{P} 7\left(\hat{\mu}_{1}, \hat{\mu}_{2} ; v_{1}^{*}, v_{2}^{*}\right)$, and an optimal solution to P8 $\left(\hat{\mu}_{1}, \hat{\mu}_{2} ; v_{1}^{*}, v_{2}^{*}\right)$ are shown in Figure 1.

\subsection{An interactive algorithm}

Figure 1 shows that if an optimal solution $\left(\mathbf{x}^{*}, \mathbf{y}^{*}, p^{*}, q^{*}, \sigma_{1}^{*}, \sigma_{2}^{*}\right)$ to $\mathrm{P} 8\left(\hat{\mu}_{1}, \hat{\mu}_{2} ; v_{1}^{*}, v_{2}^{*}\right)$ satisfies the equality conditions (39), (40), then $\left(\mathbf{x}^{*}, \mathbf{y}^{*}, v_{1}^{*}, v_{2}^{*}\right)$ is an equilibrium solution to $\mathrm{P} 6\left(\hat{\mu}_{1}, \hat{\mu}_{2}\right)$. Unfortunately, we cannot specify such parameters $\left(v_{1}^{*}, v_{2}^{*}\right)$ in advance. On the other hand, since the first term $\mathbf{x}^{T} A_{k, v_{1}^{*}+\hat{\mu}_{1 k}} \mathbf{y}$ in the left hand of the inequality constraint (43d) is strictly monotone decreasing with respect to $v^{*}$, and the second term $\mu_{\tilde{G}_{1 k}}^{-1}\left(v_{1}^{*}+\hat{\mu}_{1 k}\right)$ in the left hand of the inequality constraint (43d) is strictly monotone 


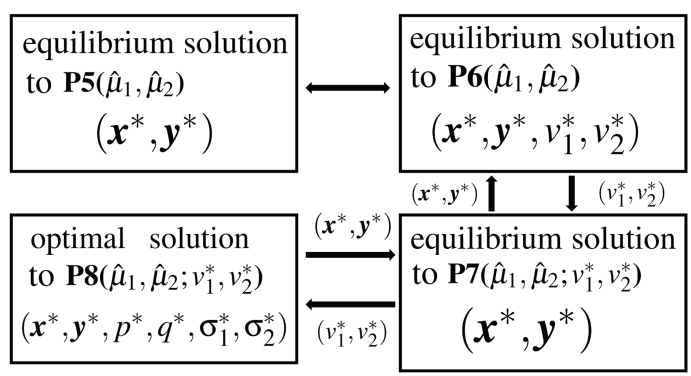

Figure 1: The relationships between P6 $\left(\hat{\mu}_{1}, \hat{\mu}_{2}\right)$, $\mathrm{P} 7\left(\hat{\mu}_{1}, \hat{\mu}_{2} ; v_{1}^{*}, v_{2}^{*}\right)$, and P8 $\left(\hat{\mu}_{1}, \hat{\mu}_{2} ; v_{1}^{*}, v_{2}^{*}\right)$

increasing with respect to $v^{*}$, there exists some value of $v_{1}^{*}$ such that $\mathbf{x}^{T} A_{k, v_{1}^{*}+\hat{\mu}_{1 k}}^{R} \mathbf{y}=\mu_{\tilde{G}_{1 k}}^{-1}\left(v_{1}^{*}+\hat{\mu}_{1 k}\right)$. At this value of $v^{*}, \sigma_{1}^{*}=0$. In a similar way, we can fined $v_{2}^{*}$ such that $\sigma_{2}^{*}=0$.

From such a point of view, we can develop the algorithm to find the values of $\left(v_{1}^{*}, v_{2}^{*}\right)$ such that $\sigma_{1}^{*}=$ $0, \sigma_{2}^{*}=0$ by updating $\left(v_{1}^{*}, v_{2}^{*}\right)$ sequentially, in which the conditions (39), (40) are satisfied. From the property of the level sets for fuzzy numbers, the parameters $\left(v_{1}^{*}, v_{2}^{*}\right)$ must satisfy the following inequalities, which depend on the reference membership values $\left(\hat{\mu}_{1}, \hat{\mu}_{2}\right)$.

$$
\begin{aligned}
& 0 \leq v_{1}^{*}+\hat{\mu}_{1 k} \leq 1, k=1, \ldots, K \\
& 0 \leq v_{2}^{*}+\hat{\mu}_{2 l} \leq 1, l=1, \ldots, L
\end{aligned}
$$

From the above conditions, the search range of $\left(v_{1}, v_{2}\right)$ is as follows.

$$
\begin{aligned}
& \max _{k=1, \ldots, K}\left(-\hat{\mu}_{1 k}\right) \leq v_{1}^{*} \leq \min _{k=1, \ldots, K}\left(1-\hat{\mu}_{1 k}\right) \\
& \max _{l=1, \ldots, L}\left(-\hat{\mu}_{2 l}\right) \leq v_{2}^{*} \leq \min _{l=1, \ldots, L}\left(1-\hat{\mu}_{2 l}\right)
\end{aligned}
$$

Using the bisection method with respect to $\left(v_{1}^{*}, v_{2}^{*}\right)$, we can find the values of $\left(v_{1}^{*}, v_{2}^{*}\right)$ such that $\sigma_{1}^{*}=\sigma_{2}^{*}=0$.

According to the above discussions, we propose an interactive algorithm to obtain a satisfactory solution of Player 1 from among an equilibrium solution set (Definition 4) under the assumption that Player 1 can estimate Player 2's reference membership values.

\section{Interactive algorithm 2}

Step 1 Each player elicit his/her membership functions $\mu_{\tilde{G}_{1 k}}(\cdot), k=1, \cdots, K, \mu_{\tilde{G}_{2 l}}(\cdot), l=1, \cdots, L$ for fuzzy expected payoffs $\mathbf{x}^{T} \tilde{A}_{k} \mathbf{y}, k=1, \cdots, K$, $\mathbf{x}^{T} \tilde{B}_{l} \mathbf{y}, l=1, \cdots, L$.

Step 2 Set Player 1's reference membership values as $\hat{\mu}_{1 k}=1, k=1, \ldots, K$. Estimate Player 2's reference membership values $\hat{\mu}_{2 l}, l=1, \ldots, L$.
Step 3 Set the initial values of the parameter $\left(v_{1}^{*}, v_{2}^{*}\right)$ as follows.

$$
\begin{aligned}
& v_{1}^{*} \leftarrow\left\{\max _{k=1, \ldots, K}\left(-\hat{\mu}_{1 k}\right)+\min _{k=1, \ldots, K}\left(1-\hat{\mu}_{1 k}\right)\right\} / 2 \\
& v_{2}^{*} \leftarrow\left\{\max _{l=1, \ldots, L}\left(-\hat{\mu}_{2 l}\right)+\min _{l=1, \ldots, L}\left(1-\hat{\mu}_{2 l}\right)\right\} / 2
\end{aligned}
$$

Step 4 Solve P8 $\left(\hat{\mu}_{1}, \hat{\mu}_{2} ; v_{1}^{*}, v_{2}^{*}\right)$, and obtain the optimal solution $\left(\mathbf{x}^{*}, \mathbf{y}^{*}, p^{*}, q^{*}, \sigma_{1}^{*}, \sigma_{2}^{*}\right)$.

Step 5 If $\sigma_{1}^{*}>0$, then $v_{1}^{*} \leftarrow v_{1}^{*}+\Delta v_{1}$, else if $\sigma_{1}^{*}<0$, then $v_{1}^{*} \leftarrow v_{1}^{*}-\Delta v_{1}$. If $\sigma_{2}^{*}>0$, then $v_{2}^{*} \leftarrow v_{2}^{*}+$ $\Delta v_{2}$, else if $\sigma_{2}^{*}<0, v_{2}^{*} \leftarrow v_{2}^{*}-\Delta v_{2}$, where $\Delta v_{1}$ and $\Delta v_{2}$ are sufficiently small positive constants, and return to Step 4. If $\sigma_{1}^{*}=0$ and $\sigma_{2}^{*}=0$ (this means that the equality conditions (39), (40) are satisfied), then go to Step 6.

Step 6 If Player 1 is not satisfied with the current values of the membership functions $\mu_{\tilde{G}_{1 k}}\left(\mathbf{x}^{* T} \hat{A}_{k} \mathbf{y}^{*}\right), k=1, \ldots, K$, then update the reference membership values $\hat{\mu}_{1 k}, k=1, \ldots, K$ and return to Step 3. Otherwise, stop.

\section{A numerical example}

To show the efficiency of the proposed algorithm, consider a situation in which two competing firms plan to release a new product, which was formulated as an uncertain bimatrix game by Gao [3]. Assume that each firm has only two marketing alternatives. A mixed strategy determines their budget among two marketing alternatives. Because of the lack of past statistical data about the demands, two kinds of indexes with respect to the demands are expressed as the following fuzzy payoff matrices.

$$
\begin{aligned}
\tilde{A}_{1} & =\left[\begin{array}{ll}
(120,40,40)_{L R} & (216,50,50)_{L R} \\
(192,42,42)_{L R} & (96,21,21)_{L R}
\end{array}\right] \\
\tilde{A}_{2} & =\left[\begin{array}{ll}
(50,20,20)_{L R} & (90,30,30)_{L R} \\
(32,15,15)_{L R} & (100,40,40)_{L R}
\end{array}\right] \\
\tilde{B}_{1} & =\left[\begin{array}{cc}
(120,30,30)_{L R} & (24,10,10)_{L R} \\
(48,20,20)_{L R} & (96,25,25)_{L R}
\end{array}\right] \\
\tilde{B}_{2} & =\left[\begin{array}{ll}
(50,20,20)_{L R} & (77,25,25)_{L R} \\
(30,10,10)_{L R} & (15,5,5)_{L R}
\end{array}\right]
\end{aligned}
$$

Each element of the above matrices are LR fuzzy numbers denoted as $\left(a_{k i j}, \alpha_{k i j}, \beta_{k i j}\right)_{L R}$ and $\left(b_{l i j}, \gamma_{l i j}, \delta_{l i j}\right)_{L R}$, respectively. $L(x)$ and $R(x)$ are set as $\max (0,1-x), x \in$ $[0,1]$. Let us apply the proposed algorithm (Interactive algorithm 2) to this example. At Step 1, hypothetical players set their membership functions as follows.

$$
\mu_{\tilde{G}_{11}}(s)=\frac{s-0}{230-0}, \quad \mu_{\tilde{G}_{12}}(s)=\frac{s-0}{110-0}
$$


Table 1: Interactive processes

\begin{tabular}{|c|c|c|c|}
\hline Iteration & 1 & 2 & 3 \\
\hline$\hat{\mu}_{11}$ & 1 & 0.8 & 0.75 \\
\hline$\hat{\mu}_{12}$ & 1 & 0.45 & 0.55 \\
\hline$x_{1}$ & 0.322157 & 0.322293 & 0.322215 \\
\hline$x_{2}$ & 0.677843 & 0.677707 & 0.677785 \\
\hline$y_{1}$ & 0.643082 & 0.932759 & 0.778314 \\
\hline$y_{2}$ & 0.356918 & 0.067241 & 0.221686 \\
\hline${ }^{*}\left(\tilde{G}_{11}\right)$ & 0.725596 & 0.765352 & 0.744281 \\
\hline$\Pi_{\mathbf{x}^{*} T} \tilde{A}_{\mathbf{y}^{*}}\left(\tilde{G}_{12}\right)$ & 0.617737 & 0.466793 & 0.548942 \\
\hline$\Pi_{\mathbf{x}^{* T} \tilde{B}_{1} \mathbf{y}^{*}}\left(\tilde{G}_{21}\right)$ & 0.54552 & 0.5452 & 0.54536 \\
\hline$\Pi_{\mathbf{x}^{*} T} \tilde{B}_{\mathbf{y}^{*}}\left(\tilde{G}_{22}\right)$ & 0.472824 & 0.479624 & 0.47601 \\
\hline
\end{tabular}

$$
\mu_{\tilde{G}_{21}}(t)=\frac{t-0}{150-0}, \quad \mu_{\tilde{G}_{22}}(t)=\frac{t-0}{90-0}
$$

At Step 2, Player 1 sets his/her own reference membership values as $\left(\mu_{11}, \mu_{12}\right)=(1,1)$, and estimates Player 2 's reference membership values as $\left(\mu_{21}, \mu_{22}\right)=(1,1)$. At Step 5, the step sizes are set as $\Delta v_{1}=\Delta v_{2}=$ 0.001. The interactive process under the hypothetical player (Player 1) is shown in Table1. At Iteration 1 , corresponding to the reference membership values $\left(\mu_{11}, \mu_{12}\right)=(1,1)$, Player 1 obtains the equilibrium solution to P5 $\left(\hat{\mu}_{1}, \hat{\mu}_{2}\right)$. To improve $\Pi_{\mathbf{x}^{* T} \tilde{A}_{1} \mathbf{y}^{*}}\left(\tilde{G}_{11}\right)$ at the expense of $\Pi_{\mathbf{x}^{* T} \tilde{A}_{2} \mathbf{y}^{*}}\left(\tilde{G}_{12}\right)$, Player 1 updates his/her reference membership values as $\left(\mu_{11}, \mu_{12}\right)=(0.8,0.45)$. In this example, at the third iteration, the satisfactory solution of Player 1 is obtained.

\section{Conclusion}

In this paper, an interactive decision making method for multiobjectve bimatrix games with fuzzy payoffs have been proposed to obtain a satisfactory solution from among an equilibrium solution set by updating the reference membership values. Until now, any method to obtain the equilibrium solution based on possibility measure for multiobjectve bimatrix games with fuzzy payoffs has not been proposed because of the bilinear fractional forms such as (26) and (26). To circumvent the computational difficulties to obtain an equilibrium solution, the equilibrium conditions in the membership function space are transformed into the equilibrium conditions in the expected payoff space. By applying the bisection method in the expected payoff space, an equilibrium solution is obtained. In the bisection method, if the step sizes $\Delta v_{1}$ and $\Delta v_{2}$ are set as large positive values, any equilibrium solution cannot be obtained, and, if the step sizes $\Delta v_{1}$ and $\Delta v_{2}$ are set as too small positive values, it will take time to converge. Although the proposed algorithm does not guar- antee to necessarily obtain any equilibrium solution, we can always obtain the approximate equilibrium solution. Moreover, even if membership functions are nonlinear, it will be possible to obtain any equilibrium solution. In the near future, we will investigate such a case.

\section{Acknowledgement}

The authors wish to thank Mr. Kanta Tsuchiya who works at Nagoya City Hall in Japan.

\section{References}

[1] H. W. Corley, Games with vector payoffs, Journal of Optimization Theory and Applications 47 (1985) 491-498.

[2] D.Dubois, H.Prade, Fuzzy Sets and Systems : Theory and Applications, Academic Press, 1980.

[3] J. Gao, Uncertain bimatrix game with applications, Fuzzy Optimization and Decision Making 12 (2013) 65-78.

[4] B. Liu, Uncertainty Theory (2nd ed.), Springer, Berlin, 2007.

[5] T. Maeda, Characterization of the equilibrium strategy of the bimatrix game with fuzzy payoff, Journal of Mathematical Analysis and Applications 251 (2000) 885-896.

[6] Z. Makó, J. Salamon, Correlated equilibrium of games in fuzzy environment, Fuzzy Sets and Systems 398 (2020) 112-127.

[7] I. Nishizaki, T. Notsu, Nondominated equilibrium solutions of a multiobjective two-person nonzero-sum game and corresponding mathematical programming problem, Journal of Optimization Theory and Applications 135 (2007) 217239.

[8] I. Nishizaki, M. Sakawa, Equilibrium solutions for multiobjective bimatrix games incorporating fuzzy goals, Journal of Optimization Theory and Applications 86 (1995) 433-458.

[9] M. Sakawa, Fuzzy sets and interactive multiobjective optimization, Plenum Press, New York, 1993.

[10] M. Tang, Z. Li, A novel uncertain bimatrix game with hurwicz criterion, Soft Computing 24 (2020) 2441-2446.

[11] P. L. Yu, Multiple-Criteria Decision Making, Concepts, Techniques, and Extensions, Plenum Press, New York, 1985. 